\title{
TECNOLOGIAS GEOESPACIAIS NO GERENCIAMENTO DA CULTURA DA MAÇÃ $\tilde{n}^{1}$
}

\author{
BERNARDO FRIEDRICH THEODOR RUDORFF², LUIGI CARLI MARRONI AULICINO², MAURICIO ALVES MOREIRA²
}

RESUMO - No presente trabalho, foram utilizadas tecnologias geoespaciais visando a auxiliar o gerenciamento e o manejo da cultura da maçã. Um GPS de navegação foi utilizado para delimitar 201 quadras de maçã na Fazenda Rio Verde situada no município de Fraiburgo-SC. As coordenadas dos pontos (waypoints) foram introduzidas num sistema de informações geográficas (SIG), obtendo-se um mapa com a distribuição dos limites das quadras de maçã. Estes limites foram associados a um banco de dados contendo informações cadastrais, tais como: variedade, data de plantio e área. Imagens do sensor ETM+ do satélite Landsat-7, adquiridas em 07 de janeiro de 2000 e 05 de agosto de 2001, foram utilizadas para mapear o uso e ocupação do solo nas áreas restantes da fazenda. O tamanho das quadras de maçã variou entre 0,14 e 5,32 ha. Uma comparação entre a área das quadras estimada pelo GPS de navegação e a área estimada a partir do número de plantas, multiplicado pela área ocupada por planta, apresentou um coeficiente de correlação r=0,97. As classes de uso e ocupação do solo foram: açude, banhado, mato, capoeira, lavoura e reflorestamento. De acordo com os resultados alcançados nesta pesquisa, pode-se chegar às seguintes conclusões: a) o uso do GPS de navegação mostrou-se viável para a obtenção do mapa com o limite das quadras de maçã; b) as imagens do Landsat permitiram identificar as diferentes classes de uso e ocupação do solo; c) o SIG associado a um banco de dados é uma importante ferramenta de gerenciamento das atividades da fruticultura em quadras.

Termos para Indexação: maçã, Sistema de Informações Geográficas, SIG, sensoriamento remoto

\section{GEOSPATIAL TECHNOLOGIES ON APPLE ORCHARDS MANAGEMENT}

\begin{abstract}
Geospatial technologies were used in the present work in order to assist apple orchards management. A navigation GPS was used to obtain waypoints for 201 apple fields in Rio Verde farm, located in the municipality of Fraiburgo, Santa Catarina State. These waypoints were introduced in the Geographical Information System (GIS) to obtain a map with the geographic limits of apple fields. These fields were then associated to a data base with several information such as: variety, planting date, area etc. Landsat-7 ETM+ images acquired on January $07^{\text {th }} .2000$ and August $05^{\text {th }} 2001$ were used to classify the land use in the remaining areas of the farm. The size of the apple fields varied from 0.14 ha to $5.32 \mathrm{ha}$. A comparison between the area estimated with the GPS and the area estimated based on the number of plants times the area of each plant per field presented a correlation coefficient of 0.97 . The classes of land used were: water bodies, swamp, forest, forest regrowth and reforestation. According to the results the following conclusions could be drawn: a) the navigation GPS appears to be an appropriate tool to obtain the geographic limits of apple fields; b) the Landsat images were useful to identify several classes of land used in the farm; c) the GIS associated to a data base is an important management tool for several apple orchards activities.
\end{abstract}

Index Terms: apple, Geographic Information Systems, GIS, remote sensing

\section{INTRODUÇÃO}

Atualmente, o Brasil é praticamente auto-suficiente na produção de maçã e ainda exporta cerca de $10 \%$ da produção para mercados altamente exigentes, como a Europa e os Estados Unidos. Até 1970, o Brasil era o maior importador de maçã do Hemisfério Sul e o $5^{\circ}$ no "ranking" mundial. A produção nacional de maçã na safra de 2001 foi de cerca de 900 mil toneladas e o Estado de Santa Catarina é responsável por aproximadamente $54 \%$ desta produção, sendo que $66 \%$ da produção do Estado vem do município de Fraiburgo, onde se encontram grandes empresas do agronegócio da pomicultura (http://www.fischerfraiburgo.com.br/ producp.htm).

Dentre elas, a Empresa Pomifrai Fruticultura S/A (POMIFRAI) estabeleceu um contato com o Instituto Nacional de Pesquisas Espaciais (INPE), no início de 2000, no sentido de verificar a possibilidade de utilizarse os recentes avanços tecnológicos nas áreas de sensoriamento remoto, Sistema de Informações Geográficas (SIG) e GPS (Global Positioning System) para auxiliar no gerenciamento informatizado das lavouras de maçã. Essa empresa possui duas fazendas: a Bom Futuro e a Rio Verde. A primeira é mais antiga na atividade da pomicultura e foi amplamente estudada por Braga (1995) sob o ponto de vista da utilização das técnicas de sensoriamento remoto e SIG, disponíveis na primeira metade da década de 1990. Já a Fazenda Rio Verde não possuía informações georreferenciadas das subparcelas (quadras) e o manejo da cultura não vinha beneficiandose da facilidade de utilização de um SIG. Com a melhora significativa do sinal dos satélites para o GPS de navegação, a partir de maio de 1999, o principal objetivo deste trabalho consistiu em utilizar este tipo de GPS para gerar o mapa com a distribuição dos limites das quadras de maçã nessa fazenda, de forma rápida, a baixo custo e com facilidade de atualização. Outro objetivo foi mapear o uso e ocupação do solo através de imagens do sensor ETM+ do Landsat-7 nas demais áreas não levantadas pelo GPS, uma vez que a identificação de áreas cultivadas com maçã atra- vés de imagens do Landsat, especialmente em lavouras novas, é pouco confiável (Provesi et al., 1986). Também foi verificada a possibilidade de realizar-se as etapas de geoprocessamento, utilizando um aplicativo de domínio público, com um breve treinamento para os técnicos da Empresa Pomifrai Fruticultura S/A.

\section{MATERIALEMÉTODOS}

A área de estudo está localizada em parte do município de Fraiburgo, no Oeste do Estado de Santa Catarina, na região do vale do Rio do Peixe e compreende a Fazenda Rio Verde, de propriedade da Empresa Pomifrai Fruticultura S/A (POMIFRAI). Informações detalhadas sobre o município, clima e solos podem ser obtidas em Braga (1995), cujo trabalho se encontra disponível na Internet <http://www.eps.ufsc.br/teses/braga/ >. A Fazenda Rio Verde tem cerca de 1.010 ha, sendo que, aproximadamente, 340 ha são de pomáceas. Os pomares de maçã da POMIFRAI estão divididos em cinco setores, onde o setor 5 corresponde à Fazenda Rio Verde. Os setores, por sua vez, estão divididos em parcelas identificadas por três algarismos, onde o primeiro algarismo se refere ao setor e os dois seguintes à parcela. Cada parcela é dividida em subparcelas denominadas de quadras. Na quadra, as informações de número de plantas, espaçamento, ano de plantio, variedade, entre outras, podem ser obtidas. Para a empresa, é importante associar as atividades de manejo e gerenciamento à quadra.

Para realizar as etapas de geoprocessamento e processamento de imagens, foi escolhido o aplicativo SPRING, que é um SIG com funções de processamento de imagens, análise espacial, modelagem numérica de terreno e consulta a bancos de dados espaciais (Câmara et al., 1996). Este aplicativo é de domínio público e encontra-se disponível na Internet (http:/ /www.dpi.inpe.br/spring.html).

Para a obtenção dos limites das quadras, utilizou-se um GPS de navegação modelo Garmin II Plus dotado de um cabo e antena com supor-

(Trabalho 134/2002). Recebido: 18/07/2002. Aceito para publicação: 31/03/2003.

${ }^{2}$ Instituto Nacional de Pesquisas Espaciais - INPE, Divisão de Sensoriamento Remoto - DSR. Av. dos Astronautas, 1.758 - CEP: $12.227-010$ - São José dos CamposSP, Brasil. e-mail: [bernardo, luigi e mauricio]@ltid.inpe.br. 
te magnético a fim de fixar a antena fora do veículo, possibilitando melhor captação do sinal dos satélites. Cada quadra foi contornada através de uma viatura (Toyota), obtendo-se os pontos com as coordenadas geográficas (waypoints) ao longo da trajetória. Foram anotados os números inicial e final dos waypoints de cada quadra para posterior associação dos pontos com as respectivas quadras. O GPS armazena até 500 waypoints, que foram transferidos para um computador na sede da empresa. Os arquivos dos waypoints, vindos do GPS em formato ASCII, foram transformados em arquivos compatíveis com o SPRING para gerar o plano de informação no SIG, contendo os limites contínuos das quadras.

As imagens do satélite foram previamente selecionadas através do catálogo de imagens Landsat 5 e 7 da Divisão de Geração de Imagens do INPE, disponível na Internet <http://www.dgi.inpe.br>. A escolha foi feita com base na disponibilidade de imagens livres de cobertura de nuvem em duas épocas distintas verão e inverno a fim de permitir uma melhor discriminação dos alvos em função da sua resposta espectral diferenciada nestes dois períodos. As imagens selecionadas foram do sensor ETM+ do satélite Landsat-7 da órbita 221 e ponto 79, adquiridas em 07 de janeiro de 2000 e 05 de agosto de 2001 (Figura 1). Estas imagens foram registradas com auxílio da carta topográfica na escala de 1:100.000 de Leblon Régis (Folha SG-22-Z-A-IV) do Instituto Brasileiro de Geografia e Estatística (IBGE). Posteriormente, as imagens foram restauradas para a resolução espacial de 5 x 5 metros.

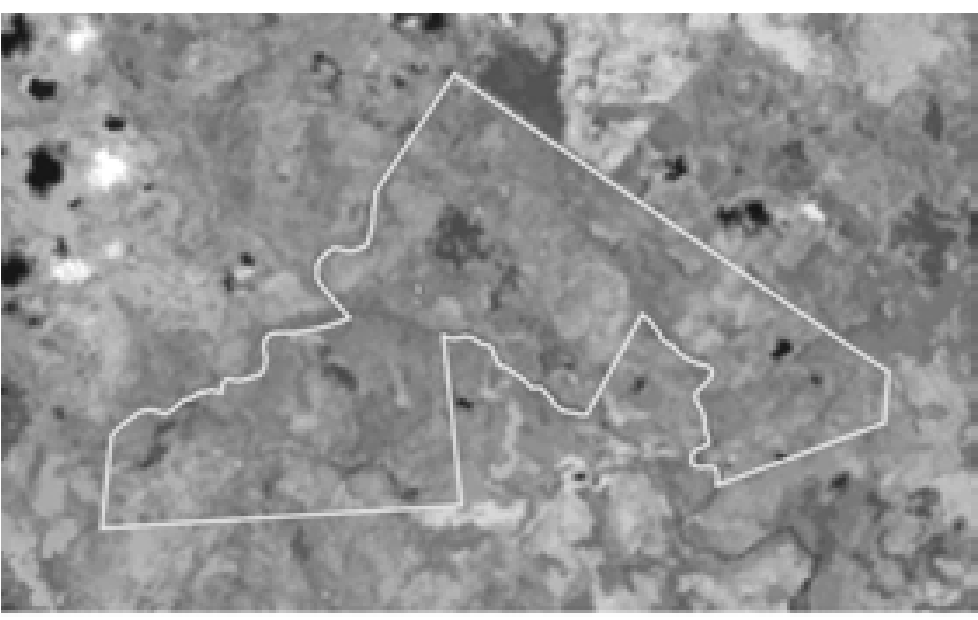

07 de janeiro de 2000

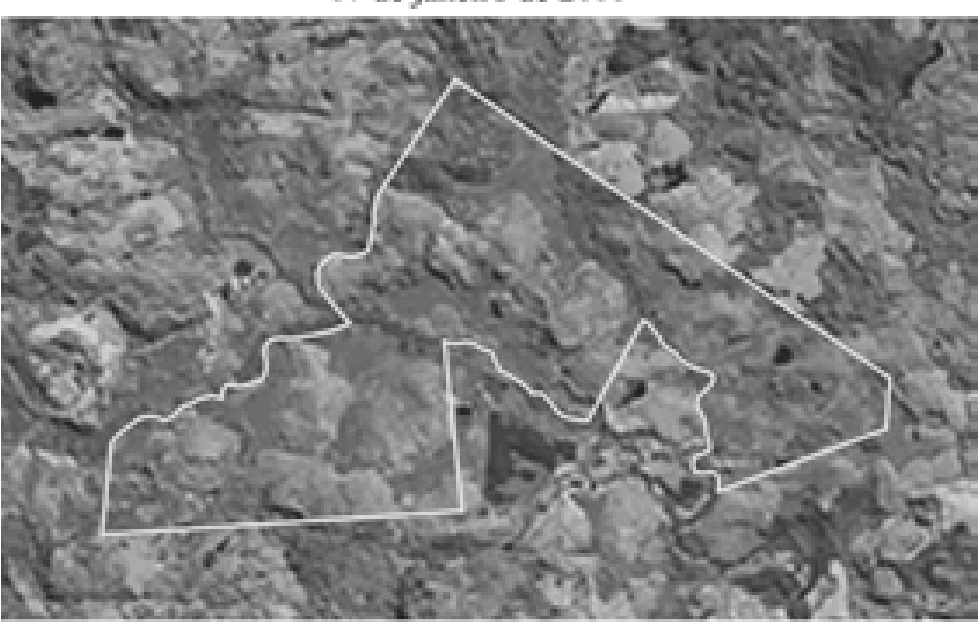

05 de agosto de 2001

FIGURA 1-Limite da Fazenda Rio Verde sobre imagens obtidas pelo satélite Landsat- 7 em 07 de janeiro de 2000 e 05 de agosto de 2001, em composição colorida das bandas 3 (azul), 4 (verde) e 5 (vermelho), restaurada para a resolução espacial de 5 x 5 metros.

As classes de uso e ocupação do solo mapeadas a partir das imagens do satélite foram: açude, banhado, mato, capoeira, lavoura, reflorestamento e outros. Um trabalho a campo feito pelos técnicos da POMIFRAI auxiliou na identificação das classes, especialmente as de capoeira e banhado, que nem sempre são bem definidas nas imagens. O tratamento das imagens foi feito, utilizando-se dos procedimentos de segmentação e de classificação não supervisionada, implementados no SPRING; seguido de uma interpretação visual das imagens na tela do computador através do procedimento de edição matricial. Um mapa (plano de informação temático) contendo as classes e as suas respectivas áreas em hectares, foi gerado.

Um breve treinamento de dois dias sobre o uso do aplicativo SPRING para fins de gerenciamento das atividades agrícolas na Fazenda Rio Verde, em um SIG, foi proporcionado a três técnicos da POMIFRAI ao final das atividades acima descritas.

\section{RESULTADOSEDISCUSSÃO}

Um total de 1860 waypoints foram capturados para 201 quadras, ou seja, uma média de 9,2 pontos por quadra. Estes pontos foram coletados em dois dias e meio de trabalho a campo. Descontando o tempo de traslado para a área, obtiveram-se em média 90 pontos por hora, ou seja, foram levantadas aproximadamente 10 quadras por hora. Os limites contínuos gerados a partir destes waypoints no SPRING permitiram obter os valores da área em hectares de cada quadra. O tamanho das quadras de maçã na fazenda variou de 0,14 a 5,14 ha pelo GPS e de 0,14 ha a 5,32 ha pelo espaçamento (área ocupada por cada planta multiplicado pelo número de plantas por quadra). Foi observada uma boa correlação $(r=0,97)$ entre a área estimada pelo GPS e a área estimada pelo espaçamento (Figura 2). Em 17 quadras, foi observado que o GPS subestimou a área das quadras em mais de $10 \%$ do valor da área estimada pelo espaçamento. Em 53 quadras, o GPS superestimou a área das quadras em mais de 10\%, quando comparado com a área estimada pelo espaçamento, e superestimou em 3,4\% o valor total das quadras de maçã, quando comparado com o estimado pelo espaçamento. É de esperar-se que a área do GPS apresente um valor pouco superior à área estimada pelo espaçamento, pois a obtenção dos waypoints foi feito, contornando as quadras pelo lado de fora e nem sempre foi possível acompanhar exatamente o posicionamento das plantas que definem as quadras. Uma verificação a campo seria recomendável para identificar as causas das diferenças superiores a 10\%. O levantamento dos limites através do GPS de navegação mostrou ser ágil e de baixo custo quando comparado com os demais levantamentos disponíveis, tais como: GPS diferencial, topográfico, fotografia aérea restituída, imagem de satélite de alta resolução, entre outros. Com o uso do GPS diferencial (DGPS), seria possível obter um resultado bem superior e que se faz necessário para certas aplicações (Gomes, 2001). Todavia, para a presente aplicação, pode-se afirmar que o GPS de navegação é uma ferramenta suficientemente precisa para obter o plano de informação com os limites das quadras de maçã, cujo ajuste sobre uma imagem do Landsat-7 é apresentado na Figura 3.

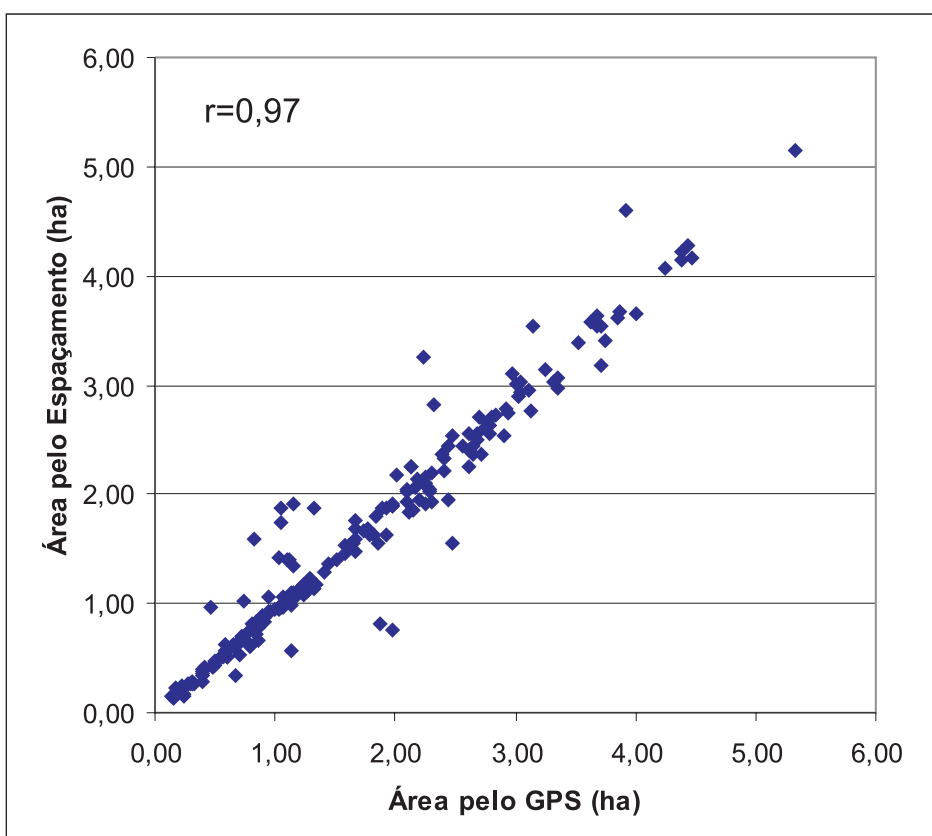

FIGURA 2-Correlação entre a área das quadras estimada pelo GPS e estimada pelo espaçamento (área ocupada por planta x número de plantas por quadra). 


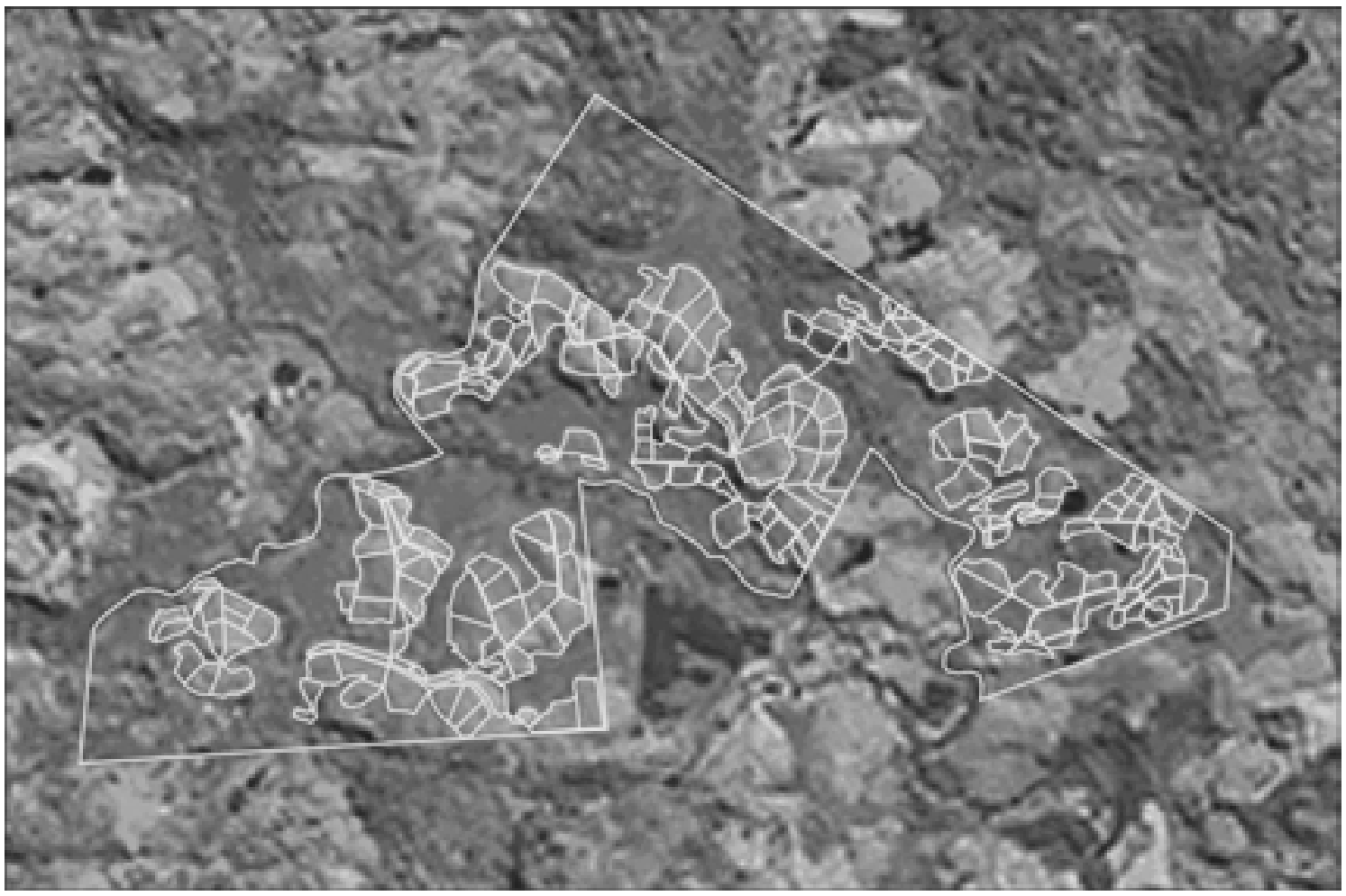

FIGURA 3- Limite das quadras de maçã obtidas pelo GPS sobrepostas à imagem ETM+ do Landsat-7 adquirida em 08 de agosto de 2001.

Após a geração deste plano de informação, foi criado um banco de dados no SPRING associando-se as informações contidas numa planilha eletrônica ao dado espacial, ou seja, ao limite de cada quadra (Figura 4).

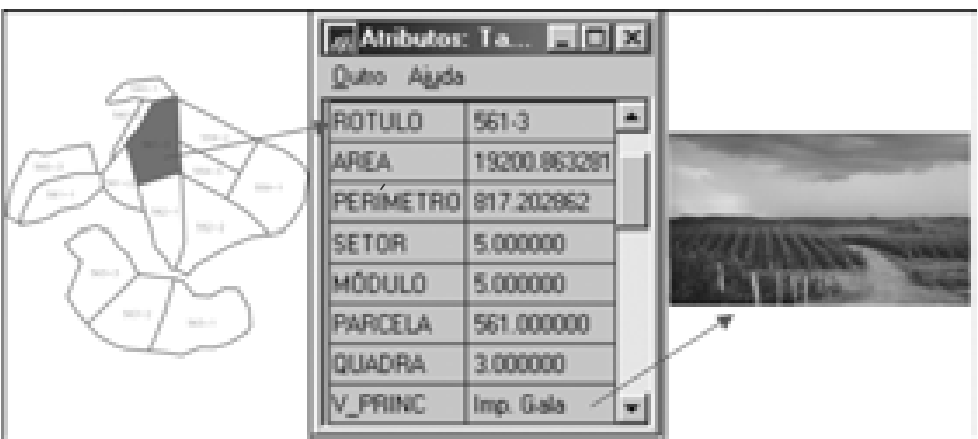

FIGURA 4- Associação da informação espacial (quadra) com o banco de dados.

A Figura 5 apresenta o mapa de uso e ocupação do solo gerado a partir das imagens do Landsat-7 para os temas banhado, capoeira, mato, reflorestamento, lavoura com cultura temporária, açude; e do GPS para o tema fruticultura. A Fazenda Rio Verde está dividida em sete glebas conforme é apresentado na Figura 5. A Tabela 1 apresenta os valores da área em hectares para os temas por gleba e total.

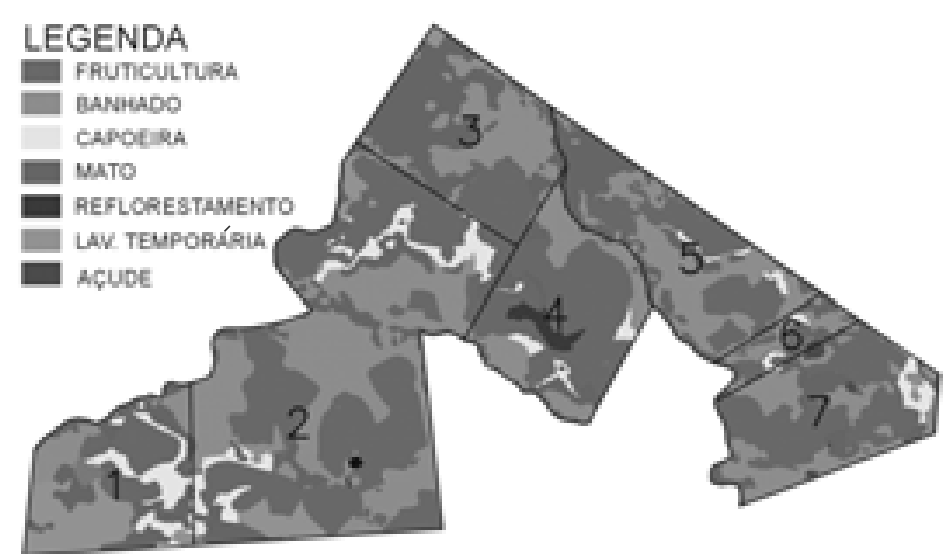

FIGURA 5 - Mapa temático da Fazenda Rio Verde.
TABELA 1-Valores da área em hectares dos temas da Fazenda Rio Verde.

\begin{tabular}{|c|c|c|c|c|c|c|c|c|}
\hline Temas & \multicolumn{7}{|c|}{ Gleba } & $\begin{array}{c}\text { Total } \\
\text { Tema }\end{array}$ \\
\hline & 1 & 2 & 3 & 4 & 5 & 6 & 7 & \\
\hline Fruticultura & 23,3 & 160,9 & 21,4 & 67,4 & 38,9 & 7,9 & 55,6 & 375,4 \\
\hline Banhado & 13,8 & 128,3 & 40,0 & 29,2 & 45,8 & 9,8 & 39,6 & 306,5 \\
\hline Capoeira & 15,2 & 22,7 & - & 5,4 & 2,5 & 1,5 & 5,4 & 52,7 \\
\hline Mato & 28,3 & 41,4 & 60,1 & 13,3 & 35,1 & 5,5 & 18,2 & 201,9 \\
\hline Reflorestamento & - & - & - & 5,9 & - & - & - & 5,9 \\
\hline Lav. Temporária & 29,0 & 16,6 & 1,5 & - & - & - & - & 47,1 \\
\hline Açude & - & 0,3 & - & 0,1 & - & 0,4 & 2,0 & 2,8 \\
\hline Outros & 2,6 & 7,5 & 0,2 & 1,4 & 1,2 & 0,4 & 2,5 & 15,8 \\
\hline Total da Gleba & 112,2 & 377,7 & 123,2 & 122,7 & 123,5 & 25,5 & 123,3 & 1008,1 \\
\hline
\end{tabular}

\section{CONCLUSÕES}

Foi observado um bom ajuste do plano de informação dos limites das quadras de maçã sobre a imagem do satélite Landsat-7, indicando ser viável o uso do GPS de navegação no levantamento do limite das quadras de maçã. Além disso, o procedimento de obtenção dos waypoints é simples e rápido. As imagens do satélite Landsat-7 foram úteis no levantamento temático do uso e ocupação do solo, fornecendo a distribuição espacial e a área de cada tema. O aplicativo SPRING, de domínio público, permitiu realizar todas as atividades de processamento de imagem, banco de dados e Sistema de Informações Geográficas utilizadas neste trabalho.

\section{REFERÊNCIAS BIBLIOGRÁFICAS}

BRAGA, H. J. Previsão agrícola: uma nova abordagem - uso de scanner aerotransportável e redes neurais. Florianópolis, 1995.150 p. Tese (Doutorado em Engenharia de Produção), Universidade Federal de Santa Catarina. Florianópolis - SC.

CÂMARA, G.; SOUZA, R. C. M.; FREITAS, U. M.; GARRIDO, J. C. P. SPRING: Integrating Remote Sensing and GIS with Object-Oriented Data Modelling. Computers and Graphics, v.15, n.6, p.13-22, July 1996.

GOMES, E.; PESSOA, L. M. C.; SILVAJÚNIOR, L. B. Medindo imóveis rurais com GPS. Brasília. LK - Editora e Comunicação Ltda., 2001. $134 \mathrm{p}$.

PROVESI, J. R.; VALERIANO, D. M.; KRUG, T. Estudo preliminar sobre a aplicação de processamento digital de dados TM- Landsat no mapeamento de pomares de maçã em Fraiburgo - SC. In: Simpósio Latino-Americano de Sensoriamento Remoto, I, Gramado - RS, Vol. 1, 1986: $772-784$. 